\title{
LPS antagonism reduces graft-versus- host disease and preserves graft-versus- leukemia activity after experimental bone marrow transplantation
}

\author{
Kenneth R. Cooke, ${ }^{1}$ Armin Gerbitz, ${ }^{2}$ James M. Crawford, ${ }^{3}$ Takanori Teshima, ${ }^{1}$ \\ Geoffrey R. Hill, ${ }^{4}$ Amy Tesolin, ${ }^{1}$ Daniel P. Rossignol, ${ }^{5}$ and James L.M. Ferrara ${ }^{1}$ \\ ${ }^{1}$ Departments of Internal Medicine and Pediatrics, Blood and Marrow Stem Cell Transplantation Program, University of \\ Michigan, Ann Arbor, Michigan, USA \\ ${ }^{2}$ Department of Hematology and Oncology, University of Regensburg, Regensburg, Germany \\ ${ }^{3}$ Department of Pathology, University of Florida School of Medicine, Gainesville, Florida, USA \\ ${ }^{4}$ Department of Hematology and Stem Cell Transplantation, Mater Medical Research Institute, South Brisbane, Australia \\ ${ }^{5}$ Eisai Inc., Teaneck, New Jersey, USA
}

Address correspondence to: Kenneth R. Cooke, Blood and Marrow Stem Cell Transplantation Program, University of Michigan Cancer Center, 1500 East Medical Center Drive, Ann Arbor, Michigan 48109-0942, USA. Phone: (734) 615-3149; Fax: (734) 647-9271; E-mail: krcooke@umich.edu.

Received for publication January 5, 2001, and accepted in revised form May 8, 2001.

\begin{abstract}
Acute graft-versus-host disease (GVHD) and leukemic relapse remain the two major obstacles to successful outcomes after allogeneic bone marrow transplantation (BMT). Recent studies have demonstrated that the loss of gastrointestinal tract integrity, and specifically the translocation of LPS into the systemic circulation, is critical to the induction of cytokine dysregulation that contributes to GVHD. Using a mouse BMT model, we studied the effects of direct LPS antagonism on GVHD severity and graft-versus-leukemia (GVL) activity. Administration of B975, a synthetic lipid-A analogue from day 0 to day +6 , reduced serum TNF- $\alpha$ levels, decreased intestinal histopathology, and resulted in significantly improved survival and a reduction in clinical GVHD, compared with control-treated animals. Importantly, B975 had no effect on donor T cell responses to host antigens in vivo or in vitro. When mice received lethal doses of P815 tumor cells at the time of BMT, administration of B975 did not impair GVL activity and resulted in significantly improved leukemia-free survival. These findings reveal a critical role for LPS in the early inflammatory events contributing to GVHD and suggest that a new class of pharmacologic agents, LPS antagonists, may help to prevent GVHD while preserving $\mathrm{T}$ cell responses to host antigens and GVL activity.
\end{abstract}

J. Clin. Invest. 107:1581-1589 (2001).

\section{Introduction}

Over the last several decades, allogeneic bone marrow transplantation (BMT) has emerged as an important therapeutic option for a number of malignant diseases. Specifically, allogeneic BMT is now accepted as the treatment of choice for adults with chronic myeloid leukemia (CML) and in adults and children with acute myeloid leukemia (AML) and acute lymphoid leukemia (ALL) with high-risk features or relapsed disease. The therapeutic potential of allogeneic BMT relies on the graft-versus-leukemia (GVL) effect, which eradicates residual malignant cells via immunologic mechanisms. Unfortunately, GVL effects are closely associated with graft-versus-host disease (GVHD), the major complication of allogeneic $\operatorname{BMT}(1,2)$.

The pathophysiology of GVHD is complex and involves donor $\mathrm{T}$ cell responses to host antigens, inflammatory cytokine effectors, and LPS, a component of endogenous bowel flora and a potent enhancer of cytokine release (3-6). During GVHD, cytokine dysreg- ulation results as a consequence of synergistic interactions between cells of both myeloid and lymphoid lineages (7). After transplantation, cytokines produced by donor $\mathrm{T}$ cells in response to host alloantigens "prime" monocytes and macrophages to secrete cytopathic amounts of inflammatory cytokines (e.g., TNF- $\alpha$ and IL-1) when stimulated by LPS that has leaked across a damaged intestinal mucosa and into the systemic circulation (8-11); thus, mice with GVHD are known to be exquisitely sensitive to the effects of LPS $(9,12,13)$. In accord with these findings, we have shown that BMT with donor cells resistant to LPS stimulation results in significantly less severe GVHD (14), and decontamination of the gut microflora has reduced GVHD severity in both experimental and clinical BMT studies (15-20).

Separation of the toxicity of GVHD from the beneficial GVL effects remains the major challenge to expanding the utility of allogeneic BMT as a treatment for hematologic malignancies. Depletion of T cells from the donor graft effectively prevents GVHD but results 
in the loss of GVL and enhanced leukemic relapse after both clinical and experimental BMT (21-23). An alternative approach to separate GVHD from GVL is to retain mature $T$ cells in the bone marrow graft but to protect the gastrointestinal (GI) tract and disrupt the amplification of early inflammatory cytokine cascades (23-25). Given the importance of LPS to the cytokine dysregulation associated with GVHD, we studied the effects of B975, a synthetic analog of Escherichia coli lipid $\mathrm{A}$, in a well-established mouse BMT model. These molecules are potent antagonists of LPS-induced cellular activation and act as competitive inhibitors at the cell surface that block NF-KB activation and nuclear translocation. They are active both in vitro and in vivo and are devoid of agonistic activity even at high doses (26). We hypothesized that administration of B975 early in the time course of allogeneic BMT would block the biologic response to LPS as it began to leak across the gut mucosal border and into the systemic circulation and downregulate the proinflammatory response associated with acute GVHD. Our data demonstrate that B975 significantly reduces TNF- $\alpha$ production and intestinal damage without altering donor $\mathrm{T}$ cell responses and ultimately results in a reduction of GVHD severity and preservation of GVL effects.

\section{Methods}

Mice and bone marrow transplantation. Female C57BL/ 6 (B6Ly5.1, H-2 $\left.{ }^{\mathrm{b}}, \mathrm{CD} 45.2^{+}\right)$and B6D2F1 (H-2 ${ }^{\text {bxd, }}$ $\left.\mathrm{CD} 45.2^{+}\right)$mice were purchased from the Jackson Laboratories (Bar Harbor, Maine, USA) and B6Ly-5.2 (H-2 ${ }^{\mathrm{b}}$, $\mathrm{CD} 45.1^{+}$) were purchased from the National Cancer Institute at Frederick (Frederick, Maryland, USA). Mice between the ages of 12 and 20 weeks were used for BMT and in vitro experiments. Mice were transplanted according to a standard protocol as described previously (27). Briefly, bone marrow (BM) was harvested from the femurs and tibias of donor mice. Cell mixtures of $5 \times 10^{6} \mathrm{BM}$ cells supplemented with $2 \times 10^{6}$ nylon-wool nonadherent splenic $\mathrm{T}$ cells from either syngeneic $(\mathrm{B} 6 \mathrm{D} 2 \mathrm{~F} 1)$ or allogeneic $(\mathrm{C} 57 \mathrm{BL} / 6)$ donors were resuspended in Leibovitz's L-15 medium (Life Technologies Inc., Grand Island, New York, USA) and transplanted into $\mathrm{B} 6 \mathrm{D} 2 \mathrm{~F} 1$ recipients by tail vein infusion $(0.25 \mathrm{ml}$ total volume) on day 0 . Consistent with previous results, $70 \%$ to $75 \%$ of cells obtained after nylon-wool passage were positive for CD4 or CD8 surface antigens (14). Before transplant, host mice received $14 \mathrm{~Gy}$ of total body irradiation $\left({ }^{137} \mathrm{Cs}\right.$ source $)$ delivered in two fractions separated by 3 hours to reduce gastrointestinal toxicity. Mice were subsequently housed in sterilized microisolator cages and received normal chow and autoclaved hyperchlorinated water for the first 3 weeks after BMT and filtered water thereafter. Our laboratory has studied the impact of CD45 allelic differences that exist between C57BL/6 and B6Ly5.2 mice on GVHD induction when using the irradiated B6 $\rightarrow$ model and have found no differences with respect to survival, clinical score, and target organ pathology (data not shown). These observations have allowed us to use B6Ly5.2 mice interchangeably with C57BL/6 mice for engraftment and GVL experiments as published $(23,25,27)$.

Leukemia induction. P815 (H-2 $\left.{ }^{\mathrm{d}} \mathrm{CD} 45.2\right)$ is a mastocytoma cell line derived from DBA/2 mice. In GVL experiments, B6Ly5.2 (CD45.1) mice were used as allogeneic BMT donors and 2,000 P815 tumor cells were added to the BM inoculum on day 0 . Survival was monitored daily, and the cause of each death after BMT was determined to be either GVHD or tumor by postmortem examination. Injection of 2,000 P815 cells into animals that cannot reject this tumor is uniformly lethal and results in massive tumor infiltration and enlargement of the liver and spleen with characteristic nodule formation (23). In some animals, lower-limb paralysis (from spinal infiltration) was also observed and was very specific for death from leukemia. Thus, death from P815 was defined by enlargement of the liver and spleen with macroscopic tumor nodules or hind-limb paralysis, whereas GVHD death was defined as the absence of tumor and the presence of GVHD as determined by the clinical scoring system described below. Minimal residual disease was determined in surviving animals without gross evidence of tumor using FACS analysis of the peripheral blood and spleen. Mixing experiments completed by adding graded amounts of CD $45.2^{+}$P815 cells to CD $45.1^{+}$splenocytes demonstrated that $0.2 \%$ or more P815 cells could be detected reliably using this technique.

Administration of B975. Compound B975 was obtained from Eisai Research Institute (Andover, Massachusetts, USA) as a dry lyophilized powder and was stored at $-80^{\circ} \mathrm{C}$. Vials of $\mathrm{B} 975$ were first dissolved in sterile 10 $\mathrm{mM} \mathrm{NaOH}$ at $0.15 \mathrm{ml}$ of $\mathrm{NaOH}$ per milligram of compound, heated to $50^{\circ} \mathrm{C}$ for 30 minutes, and then diluted to a final concentration of $375 \mu \mathrm{g} / \mathrm{ml}(37.5 \mu \mathrm{g} / 0.1 \mathrm{ml})$ with $5 \%$ dextrose water $\left(\mathrm{D}_{5} \mathrm{~W}\right)$. The $\mathrm{pH}$ was subsequently adjusted to 7.3-7.5, and $2 \mathrm{ml}$ aliquots of the diluted preparation were frozen at $-80^{\circ} \mathrm{C}$ until just before use. In pre-BMT experiments, naive B6D2F1 mice received $75 \mu \mathrm{g}$ of $\mathrm{B} 975$ intraperitoneally in two doses separated by 4 hours. Forty-five minutes after the second dose, serum was collected and analyzed for antagonistic activity to LPS-induced TNF- $\alpha$ release as described below. In subsequent experiments, recipients of allogeneic BMT received $75 \mu \mathrm{g}(0.2 \mathrm{ml})$ of B975 or 0.2 $\mathrm{ml}$ of $\mathrm{D}_{5} \mathrm{~W}$ intraperitoneally twice daily from day 0 to day +6 (14 possible injections), whereas all syngeneic BMT recipients received $\mathrm{D}_{5} \mathrm{~W}$ alone. On day 0 , the first injection was given before the initial dose of total-body irradiation (TBI), and the second was administered after completion of TBI and infusion of BM and T cells.

Systemic and histopathologic analysis of GVHD. The degree of systemic GVHD was assessed by a standard scoring system as described previously (13). Transplanted mice were ear punched, and individual weights were obtained and recorded on day 0 and weekly thereafter. A clinical index was generated week- 
ly by summation of five criteria scores: percentage of weight change, posture (hunching), activity, fur texture, and skin integrity (maximum index $=10$ ). We have found this index to be a more sensitive index of GVHD severity than weight loss alone, a parameter that has been found to be a reliable indicator of systemic GVHD in multiple mouse models (28).

Acute GVHD was also assessed by detailed histopathologic analysis of the small (ileum) and large (ascending) intestine. Specimens were harvested from animals on days +4 to +6 , placed in $10 \%$ buffered formalin, embedded in paraffin, cut into $5-\mu \mathrm{m}$-thick sections, and stained with hematoxylin and eosin for histological examination. Slides were coded without reference to mouse type or prior treatment status and examined systematically by a single pathologist (J.M. Crawford) using a semiquantitative scoring system as described previously (14).

Cell culture, analysis of proliferative response, and cytokine production. All culture media reagents were purchased from GIBCO BRL (Gaithersburg, Maryland, USA). For analysis of proliferative response in mixed lymphocyte cultures, splenocytes from individual animals were harvested on day +13 and suspended in $10 \%$ FCS/DMEM supplemented with $50 \mathrm{U} / \mathrm{ml}$ penicillin, $50 \mu \mathrm{g} / \mathrm{ml}$ streptomycin, $2 \mathrm{mM}$ L-glutamine, $1 \mathrm{mM}$ sodium pyruvate, 0.1 $\mathrm{mM}$ nonessential amino acid, $0.02 \mathrm{mM} \beta$-mercaptoethanol, and $10 \mathrm{mM}$ HEPES ( $\mathrm{pH}$ 7.75). These cells $\left(2 \times 10^{5}\right)$ were cultured in flat-bottomed 96-well Falcon plates (Becton Dickinson and Co., Franklin Lakes, New Jersey, USA) in the presence of irradiated (2,000 cGy) peritoneal cells obtained from naive B6D2F1 (host) animals at $37^{\circ} \mathrm{C}$ in a humidified incubator supplemented with $7.5 \% \mathrm{CO}_{2}$. Proliferative response to host antigen was measured by a 1205 Betaplate reader (Wallac Oy, Turku, Finland) after 72 hours by incorporation of $\left[{ }^{3} \mathrm{H}\right]$ thymidine $(1 \mu \mathrm{Ci}$; NEN Life Sciences Products Inc., Boston, Massachusetts, USA) for the last 24 hours of incubation.

For studies of TNF- $\alpha$ secretion, peritoneal cells from naive $\mathrm{B} 6 \mathrm{D} 2 \mathrm{~F} 1$ animals were suspended in 10\% FCS DMEM supplemented as above and plated at $2 \times 10^{5}$ cells per well in flat-bottomed 96-well Falcon plates (Becton Dickinson and Co.) with $10 \mathrm{ng} / \mathrm{ml}$ of LPS or media alone. Cell culture was performed at $37^{\circ} \mathrm{C}$ in a humidified incubator supplemented with $7.5 \% \mathrm{CO}_{2}$. Naive serum, serum collected after B975 injection, or 1 $\mu \mathrm{M}$ B975 were added to some wells in order to measure the extent of LPS antagonism. Supernatants were collected after 4 hours of culture and analyzed for TNF- $\alpha$ levels by using ELISA as described below.

${ }^{51} \mathrm{Cr}$ release assays. Tumor targets, $2 \times 10^{6} \mathrm{P} 815\left(\mathrm{H}-2^{\mathrm{d}}\right)$ or EL4 $\left(\mathrm{H}-2^{\mathrm{b}}\right)$, were labeled with $100 \mu \mathrm{Cu}$ of ${ }^{51} \mathrm{Cr}$ sodium salt (NEN Life Sciences Products Inc.) for 2 hours. After washing three times, labeled targets were resuspended in 10\% FCS RPMI and plated at $10^{4}$ cells per well in U-bottom plates (Corning-Costar Corp., Cambridge, Massachusetts, USA). Allogeneic splenocyte preparations as described above were added to quadruplicate wells at varying effector-to-target (effector,
$\mathrm{CD}^{+}$plus $\left.\mathrm{CD}^{+}\right)$ratios and incubated for 5 hours as above. Maximal and background release were determined by the addition of Triton-X (Sigma Chemical Co., St. Louis, Missouri, USA) or media alone to targets, respectively. ${ }^{51} \mathrm{Cr}$ activity in supernatants taken 5 hours later were determined in an auto-gamma counter (Packard Instrument Co., Meriden, Connecticut, USA), and lysis was expressed as a percentage of maximum: percentage of specific lysis $=100$ (sample count - background count/maximum count - background count).

Cell surface phenotype analysis. To analyze cell surface phenotype, splenocytes from naive or transplanted mice and P815 tumor cells were resuspended in PBS and stained with FITC conjugated mAb's to CD4, CD45.1, or CD45.2 (PharMingen, San Diego, California, USA) and phycoerythrin-conjugated (PE-conjugated) CD8 (PharMingen) for flow cytometric analysis. Cells $(0.5 \times$ $10^{6}$ ) were incubated for 20 minutes at $4^{\circ} \mathrm{C}$ with $\mathrm{mAb}$ $2.4 \mathrm{G} 2$ to block nonspecific staining via $\mathrm{Fc}$ receptors and then with the appropriate FITC- or PE-conjugated mAb's for 30 minutes at $4^{\circ} \mathrm{C}$. The cells were subsequently washed twice with PBS $/ 0.2 \%$ BSA before fixation in $1 \%$ paraformaldehyde. Two-color flow-cytometric analysis of $10^{4}$ cells was performed using a FACScan (Becton Dickinson Immunocytometry Systems, Mountain View, California, USA). The FACScan was calibrated using PE- and FITC-conjugated, nonspecific IgG Ab's.

Cytokine ELISAs. Concentrations of TNF- $\alpha$ and IFN- $\gamma$ were measured in serum and cell culture supernatants by sandwich ELISA using specific anti-mouse mAb's for capture and detection, and the appropriate standards were purchased from PharMingen (IFN- $\gamma$ ) and Genzyme Pharmaceuticals (TNF- $\alpha$; Cambridge, Massachusetts, USA). Assays were performed according to the manufacturer's protocol. Samples were diluted 1:2, and samples and standards were run in duplicate. The sensitivity of the assays was $16-20 \mathrm{pg} / \mathrm{ml}$ for TNF- $\alpha$ and $0.25 \mathrm{U} / \mathrm{ml}$ for IFN- $\gamma$. ELISA plates were read at $450 \mathrm{~nm}$ using a microplate reader (Bio-Rad Laboratories Inc., Hercules, California, USA).

Statistical considerations. All values are expressed as the mean plus or minus SEM. Statistical comparisons between groups were completed using the nonparametric, unpaired, Mann-Whitney test, except for analyzing survival data, when the Wilcoxon rank test was used.

\section{Results}

Antagonism of LPS-induced cellular activation with B975 reduces TNF- $\alpha$ production after allogeneic BMT. We first tested the effects of $\mathrm{B} 975$ in an irradiated $\mathrm{P} \rightarrow \mathrm{F} 1$ mouse model of acute GVHD. Preliminary studies using naive animals demonstrated that $\mathrm{B} 975$ was present in the serum 45 minutes after intraperitoneal injection and capable of neutralizing TNF- $\alpha$ secretion in vitro (data not shown). Lethally irradiated B6D2F1 mice received $\mathrm{BM}$ and splenic $\mathrm{T}$ cells from allogeneic (B6) or syngeneic (B6D2F1) donors as described in Methods. Allogeneic BMT recipients were injected intraperitoneally with either B975 $(75 \mu \mathrm{g})$ or control diluent twice daily from 


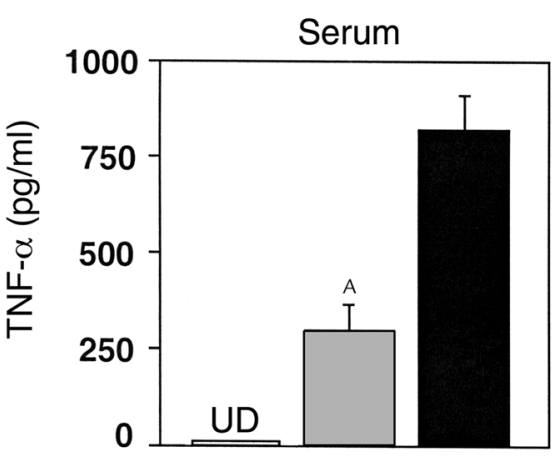

\begin{abstract}
Figure 1
Injection of B975 inhibits in vivo TNF- $\alpha$ production after BMT. Lethally irradiated $\mathrm{B} 6 \mathrm{D} 2 \mathrm{~F} 1$ received allogeneic $\mathrm{BMT}$ from $\mathrm{C} 57 \mathrm{BL} / 6$ donors and were treated with $\mathrm{B} 975$ (gray bar) or $\mathrm{D}_{5} \mathrm{~W}$ (black bar) as described in Methods. Syngeneic BMT recipients treated with $D_{5} W$ (open bar) served as negative GVHD controls. Serum was harvested between days 4 and 6 after BMT and analyzed for TNF- $\alpha$ by ELISA. Data are expressed as mean \pm SEM. ${ }^{A} P<0.05$; gray bar vs. black bar. $n=6$ to 8 per group. UD, undetectable.
\end{abstract}

day 0 to day +6 , whereas recipients of syngeneic BMT received diluent only. We have previously shown that serum endotoxin and TNF- $\alpha$ levels are significantly elevated in allogeneic BMT recipients during this time period $(11,14,27)$. We hypothesized, therefore, that this dose and schedule of B975 would neutralize LPS at a time when it begins to enter the systemic circulation and trigger TNF- $\alpha$ release. As a first screen for successful LPS antagonism after BMT, serum was obtained from day +4 to +6 and analyzed for TNF- $\alpha$. Administration of $\mathrm{B} 975$ reduced serum TNF- $\alpha$ levels by approximately $60 \%$ compared with allogeneic controls (Figure $1 ; P<0.05)$, although levels remained significantly higher compared with syngeneic BMT recipients.
Inbibition of LPS-induced cytokine secretion decreases the severity of intestinal and systemic GVHD after allogeneic BMT. TNF- $\alpha$ is known to be a critical inflammatory mediator of GI toxicity after allogeneic BMT $(14,29,30)$. Therefore we next analyzed whether the reduction in TNF- $\alpha$ levels seen after B975 administration correlated with GI pathology. Samples of small and large bowel were obtained between day +4 and +6 after BMT and were evaluated microscopically to determine an index of injury. Examination of small bowel from syngeneic BMT recipients showed reestablishment of intestinal architecture with near normal height of small bowel villi (Figure 2a) and near normal depth of colonic crypt structures. The primary feature observed was regenerative change as evidenced by increased nuclear staining and nuclear/cytoplasmic ratios. By contrast, recipients of allogeneic BMT treated with control diluent developed severe intestinal toxicity that included severe villous blunting, crypt destruction, and epithelial attenuation with intense lamina propria inflammation of the small bowel (Figure 2c). Single-cell apoptosis, although not clearly evident at the magnification shown, was maximal in the bowel of this BMT group. The intestine of allogeneic BMT recipients treated with B975 exhibited partial restoration of small bowel villi (Figure $2 b$ ) and large bowel crypts and showed evidence of regenerative change and intact epithelial layers with little inflammatory cell infiltrates. These findings and others that have been found to be associated with acute GVHD (31) were incorporated into a semiquantitative scoring system described in Methods. As shown in Figure 3a, treatment with B975 significantly reduced the small bowel pathology index compared with allogeneic controls $(P=0.02)$, although scores remained significantly higher than those observed after syngeneic BMT. Even greater differences between groups were seen in the large bowel where there was no difference between B975 and syngeneic groups (Figure $3 \mathrm{~b} ; P<0.01$ ). Because reductions
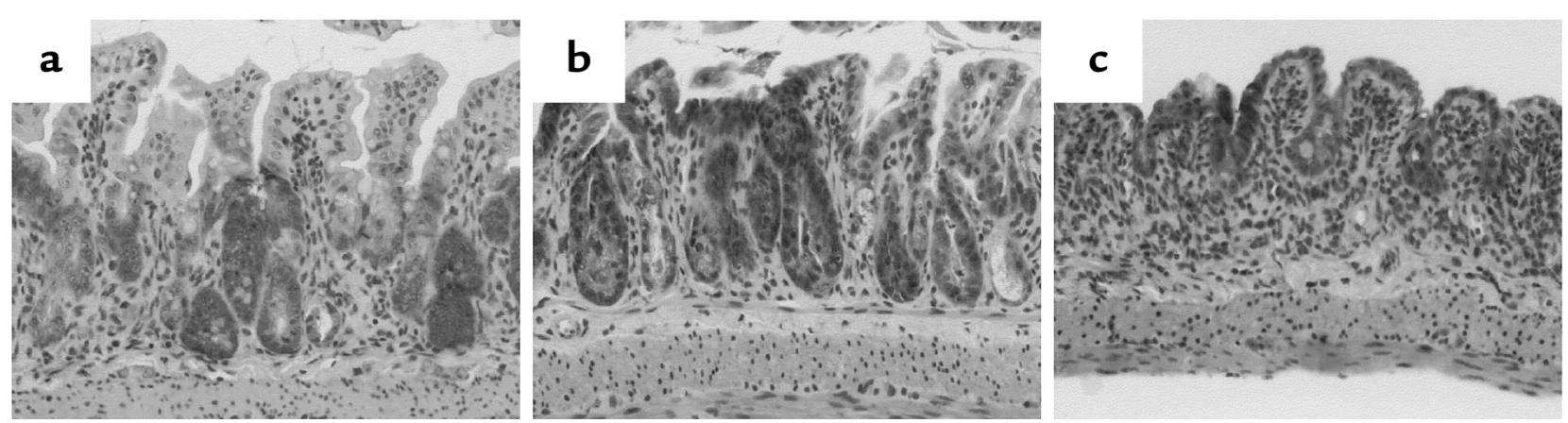

\section{Figure 2}

Treatment with B975 results in decreased intestinal histopathology after allogeneic BMT. B6D2F1 animals received syngeneic BMT or allogeneic BMT from C57BL/ 6 donors as in Figure 1. On days +4 to +6 , small-bowel samples from BMT recipient mice were obtained and analyzed microscopically as described in Methods. Syngeneic BMT recipients (a) demonstrate reestablishment of intestinal architecture with villi of near normal length and without significant cellular infiltration into the lamina propria. Regenerative change is evident by focally increased nuclear staining and an increased nuclear/cytoplasmic ratios. Animals treated with B975 (b) exhibit partial restoration of small-intestinal villous architecture, regenerative change, and little inflammatory infiltration. Recipients of allogeneic BMT (c) show severe intestinal toxicity including surface erosion, villous blunting, epithelial attenuation, and an intense cellular infiltration in the lamina propria. Original magnification, $\times 200$. 

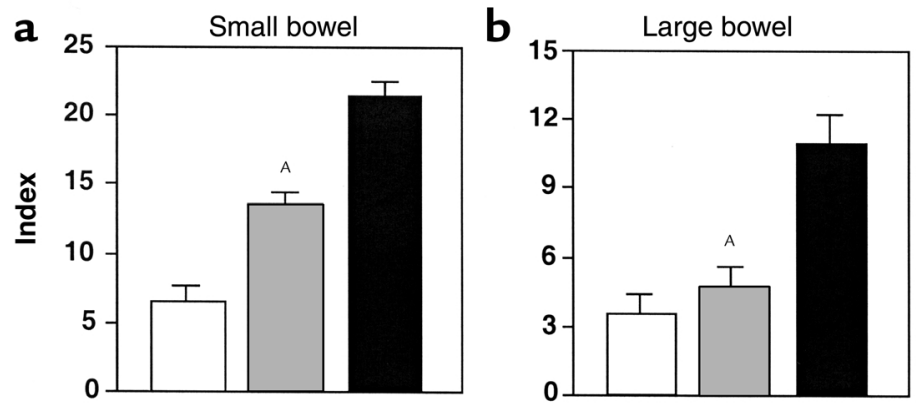

\begin{abstract}
Figure 3
Treatment with $\mathrm{B} 975$ reduces damage to the small (a) and large (b) bowel after allogeneic BMT. B6D2F1 mice received allogeneic BMT from C57BL/ 6 donors and either B975 (gray bars) or $\mathrm{D}_{5} \mathrm{~W}$ (black bars) as in Figure 1. Syngeneic BMT recipients treated with $\mathrm{D}_{5} \mathrm{~W}$ served as controls (white bars). Samples of small (a) and large (b) bowel were harvested from transplanted animals on days +4 to +6 and analyzed using the semiquantitative scoring system described in Methods. Data are expressed as mean plus or minus SEM. ${ }^{A} P<0.01$; gray barvs. black bar. $n=4$ (syngeneic) to 8 (allogeneic) per group.
\end{abstract}

in gut toxicity early in the time course of BMT have been shown to have a significant impact on overall systemic GVHD severity $(11,14)$, we next evaluated the effects of B975 on GVHD mortality and clinical score. Administration of B975 early after BMT resulted in a significant survival advantage (Figure 4a; $P<0.01$ ) and reduced the mean GVHD severity index (assessed by a clinical scoring system evaluating changes in weight, skin integrity, fur texture, posture, and mobility as described in Methods) of surviving animals compared allogeneic controls through day +35 . (Figure $4 \mathrm{~b} ; P<0.05$ ). Thus, direct antagonism of LPS with B975 significantly reduced intestinal injury and led to a reduction in clinical GVHD and a significant improvement in survival.

Effective inbibition of LPS-induced cellular activation does not impair T cell responses to host antigens after BMT. The induction of GVHD depends upon the ability of donor $T$ cells to recognize and react to antigenic disparities present in an immunocompromised host (32). Because TNF- $\alpha$ and LPS have been shown to enhance the activation, expansion, and survival of $\mathrm{T}$ cells both in vitro and in vivo
(33-35), it was possible that the effects of B975 on GVHD severity were mediated in part by diminished donor $T$ cell responses to host antigens. We therefore next examined the effects of B975 on T cell alloreactivity by evaluating $\mathrm{T}$ cell expansion, proliferation and CTL activity to host antigens and serum IFN- $\gamma$ levels. As shown in Table 1, no differences in IFN- $\gamma$ levels were observed between allogeneic groups at day +4 after BMT, when levels were significantly elevated compared with syngeneic BMT recipients $(P<$ 0.01 ). The numbers of $\mathrm{CD} 4^{+}$or $\mathrm{CD} 8^{+}$splenic lymphocyte populations were comparable on day +13 after allogeneic BMT, the time point of maximal donor $\mathrm{T}$ cell expansion in this model. Furthermore, donor T cells from B975 and diluent-treated BMT recipients proliferated equally when restimulated with irradiated host antigens in vitro and showed equivalent lysis of host type P815 $\left(\mathrm{H}-2^{\mathrm{d}}\right)$ tumor targets in a chromium release assay (Table 1). Taken together, these data suggest that $\mathrm{B} 975$ effectively reduced GVHD severity by decreasing TNF- $\alpha$ production after BMT while preserving donor $\mathrm{T}$ cell responses to host antigens.

Administration of $B 975$ preserves GVL activity after allogeneic BMT. Maintenance of donor $\mathrm{T}$ cell responses to host antigens suggested that treatment with B975 might preserve GVL activity in addition to its ability to decrease acute GVHD after allogeneic BMT. To test this hypothesis, we added 2,000 P815 (H-2d CD45.2 $\left.{ }^{+}\right)$mastocytoma cells to the BM inoculum on the day of BMT. When injected intravenously into syngeneic $\mathrm{H}-$ $2^{\text {bxd }}$ hosts, P815 was uniformly lethal by day +14 (Figure 5) and behaved like leukemia, resulting in massive infiltration and enlargement of the liver and spleen with characteristic nodule formation in all animals and hind-limb paralysis in a significant number (Table 2). Allogeneic BMT recipients again received $B 975$ or control diluent as above together with 2,000 P815 cells. As shown in Figure 5, recipients of allogeneic BMT that

$2.8 \pm 0.5$
$8.7 \pm 1.4$
$11.5 \pm 1.9$
$9449 \pm 1190$
$2349 \pm 267$
$4.0 \pm 0.5$
$36.8 \pm 1.4$
$0.9 \pm 0.2$


.
in Figure 1.
ther irradiated
ense and CTL

B6D2F1 mice received syngeneic BMT or allogeneic BMT from C57BL/ 6 mice as described in Figure 1. On day +4 , serum was harvested and analyzed for IFN- $\gamma$ by ELISA. On day +13 , splenocytes were harvested and analyzed for $T$ cell surface markers and then were restimulated in vitro with either irradiated host (B6D2F1) antigens or chromium-labeled tumor targets to measure proliferative response and CTL activity, respectively, as described in Methods. Recipients of syngeneic BMT served as negative GVHD controls. Data are expressed as mean plus or minus SD: ${ }^{A} P<0.01,{ }^{B} P<0.05$, syngeneic vs. both allogeneic groups. Resp, responder; Stim, stimulator; E/T, effector/target ratio. 


\section{Figure 4}

Treatment with B975 reduces GVHD-related mortality (a) and clinical score (b) after BMT. B6D2F1 mice received syngeneic (syn; $n=12$ ) or allogeneic (allo) BMT from C57BL/ 6 donors and either $\mathrm{B} 975(n=24)$ or $\mathrm{D}_{5} \mathrm{~W}(n=26)$ as described in Figure 1 . Transplanted animals were monitored daily for survival and assessed weekly for clinical score as described in Methods. (a) Percentage of surviving animals after BMT. Results represent a combination of three similar experiments. ${ }^{A} P<0.01$; allogeneic $+B 975$ vs. allogeneic $+D_{5} W$. (b) GVHD scores of surviving animals. Data are expressed as mean \pm SEM and represent a combination of three similar experiments. ${ }^{A} P<0.01,{ }^{B} P<0.05$; allogeneic $+B 975$ vs. allogeneic $+D_{5} W$. a Survival

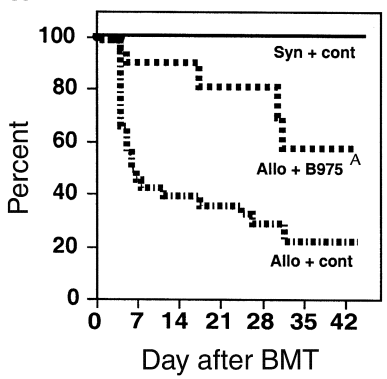

b GVHD Score

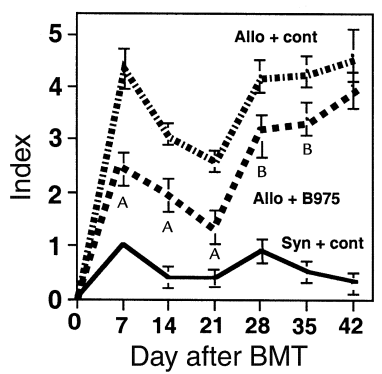

received $\mathrm{B} 975$ had a significant survival advantage compared with allogeneic controls (50\% vs. $20 \%$; $P<$ $0.05)$. Necropsy was performed on all animals either on the day of death or on day +35 to evaluate the presence of tumor. This large number of in the recipients of syngeneic BMT, did not cause hepatic or splenic enlargement in any of the allogeneic recipients (all of which had signs of acute GVHD), which is indicative of a graft-versus-tumor effect. While two of ten animals treated with $\mathrm{D}_{5} \mathrm{~W}$ versus zero of ten animals in the B975-treated group were noted to have an isolated tumor nodule in the liver, residual tumor was not identifiable by flow cytometry in the blood or spleen of any allogeneic BMT recipient tested (sensitivity $0.2 \%$ ). In a final experiment, 2,000 P815 cells were added to the $\mathrm{BM}$ inoculum of syngeneic BMT recipients that were subsequently treated with either $\mathrm{D}_{5} \mathrm{~W}$ or $\mathrm{B} 975$ from day 0 to 6 as above ( $n=8$ per group). As a survival control, some animals received syngeneic BMT without P815. Median time to death from systemic P815 tumor infiltration was 13 days in each group, and all animals died by day 14 or day 15 with evidence of massive liver $(2.41 \pm 0.3$ vs. $2.40 \pm 0.1 \mathrm{~g})$ and splenic $(0.28 \pm 0.06$ vs. $0.34 \pm 0.01 \mathrm{~g})$ enlargement in the $\mathrm{D}_{5} \mathrm{~W}$-and $\mathrm{B} 975$-treated animals respectively. Taken together, these data confirm that administration of B975 early after BMT reduces GVHD severity while preserving the beneficial GVL effect associated with allogeneic BMT without having a direct, in vivo antitumor activity.

\section{Discussion}

The results of this study suggest that direct inhibition of LPS may be an effective strategy to reduce GVHD without impairing GVL. B975 is one in a family of synthetic analogues of E. coli lipid A that are potent antagonists of LPS-induced cellular activation both in vitro and in vivo (26). We hypothesized that $\mathrm{B} 975$ would be most effective when administered the first week after BMT at a time when LPS begins to leak across a damaged gut mucosa and into the systemic circulation. Our data show that antagonism of LPS from day 0 to day +6 significantly suppressed serum TNF- $\alpha$ levels, reduced both intestinal damage and systemic GVHD, but did not alter T cell activity to host antigens either in vivo or in vitro. These allospecific donor responses likely contributed both to the persistence of the clinical GVHD observed at later time points after BMT and to the preservation of potent allogeneic GVL effects, since no animal receiving B975 showed evidence of residual leukemia.

Acute GVHD remains the most significant complication of allogeneic BMT and the major limitation to the wider application of this form of therapy. The induction of acute GVHD is a direct consequence of donor T cell responses to host alloantigens and the dysregulation of inflammatory cytokine cascades (3). Inflammatory cytokines are now known to play a central role in the pathogenesis of many transplant-related complications (36-38). In particular, TNF- $\alpha$ has been established as an important effector molecule of tissue damage resulting from BMT-conditioning regimens $(36,38,39)$ and both clinical $(37,38,40)$ and experimental $(11,14,29)$ GVHD. Specifically, TNF- $\alpha$ significantly contributes to the development of GI tract damage early after BMT. Studies completed by our group strongly suggest that the GI tract plays a major pathophysiologic role in the amplification of systemic GVHD (10). Injury to the GI tract initiated by BMT conditioning regimens allows passage of LPS into the circulation where it triggers

Table 2

P815 tumor burden after BMT

\begin{tabular}{lcccc}
\hline BMT group & Paralysis & Spleen $(\mathrm{g})$ & Liver $(\mathrm{g})$ & Nodules \\
Allogeneic $+\mathrm{D}_{5} \mathrm{~W}$ & $0 / 10$ & $0.07 \pm 0.01$ & $0.75 \pm 0.07$ & $2 / 10$ \\
Allogeneic $+\mathrm{B} 975$ & $0 / 10$ & $0.06 \pm 0.01$ & $0.75 \pm 0.07$ & $0 / 10$ \\
Syngeneic $+\mathrm{D}_{5} \mathrm{~W}$ & $3 / 8^{\mathrm{A}}$ & $0.28 \pm 0.03^{\mathrm{A}}$ & $2.33 \pm 0.16^{\mathrm{A}}$ & $8 / 8$
\end{tabular}

B6D2F1 mice received syngeneic BMT or allogeneic BMT from B6Ly5.2 donors and were treated with B975 or D $\mathrm{W}$ as in Figure 1 . P815 mastocytoma cells $(2,000)$ were added to the BM inoculum on day 0 . Animals were monitored daily for survival and evidence of hind-limb paralysis. At the time of death, or on day +35 , necropsy was performed to assess gross tumor burden. ${ }^{A} P<0.01$; syngeneic vs. both allogeneic groups. 
cytokine release by mononuclear cells that have been "primed" by IFN- $\gamma$ and propagates the "cytokine storm" characteristic of this disorder. These inflammatory mechanisms ultimately perpetuate gut mucosal damage and the translocation of additional LPS, thus establishing a positive feedback loop for progressive GI tract injury and dysfunction. In the current study, direct LPS antagonism with B975 early after BMT decreased systemic TNF- $\alpha$ levels and intestinal damage and thereby effectively blunted this deleterious feedback loop.

This conceptual framework underscores the role of LPS in the development of acute GVHD as suggested by several groups $(9,11,41,42)$. LPS is a major structural component of Gram-negative bacteria and is a potent stimulator of cellular activation and cytokine production $(5,6)$. LPS shed from bacteria that comprise normal bowel flora can elicit a broad range of inflammatory responses from macrophages, monocytes, and neutrophils. As mentioned above, these responses are significantly enhanced after allogeneic BMT due to the secretion of IFN- $\gamma$ by activated, alloreactive, $\mathrm{T}$ cells from the donor $(9,42)$. After the transplantation of allogeneic BM and T cells, LPS accumulates in both the liver and the spleen of animals with GVHD before its appearance in the systemic circulation (41). Furthermore, elevated serum levels of LPS have been shown to correlate directly with histopathologic and biochemical parameters of intestinal injury occurring during both experimental and clinical GVHD, respectively $(14,43-46)$.

Data supporting a role for LPS in this process are also provided by both experimental and clinical studies examining the effects of decontamination of the GI tract on the incidence of $\operatorname{GVHD}(17,18,47)$. Early animal studies showed that death from GVHD could be prevented if mice were give antibiotics to decontaminate the gut; normalization of the intestinal flora at or before day 20 abrogated this effect (47). In the clinical setting, Gram-negative gut decontamination has also been shown to reduce $\operatorname{GVHD}(17,18,48)$, and the intensity of this decontamination has been demonstrated recently to be an important predictor of GVHD severity $(19,20)$. Of particular interest, a study by Cohen and colleagues demonstrated that naturally occurring $\mathrm{Ab}$ titers to a rough-mutant strain of E. coli J5, which protect humans and animals from septic shock, were also associated with a decreased incidence of acute GVHD after allogeneic BMT (49). A follow-up study by the same group used a polyclonal antiserum against $E$. coli 55 as prophylaxis for acute GVHD in a prospective, double-blind, placebo-controlled trial. In this small trial, the infusion of the J5 $\mathrm{Ab}$ reduced overall GVHD from $63 \%$ to $42 \%$, and the antiserum was found to be particularly efficacious in a subset of patients with severe GVHD (50). Additional support for the role of LPS in GVHD induction comes from a study from our laboratory comparing the capacity of cells from donors that were genetically sensitive (i.e., normal) or resistant to the effects of LPS to induce GVHD. In these experiments, the donor mouse strains were otherwise identical, and,

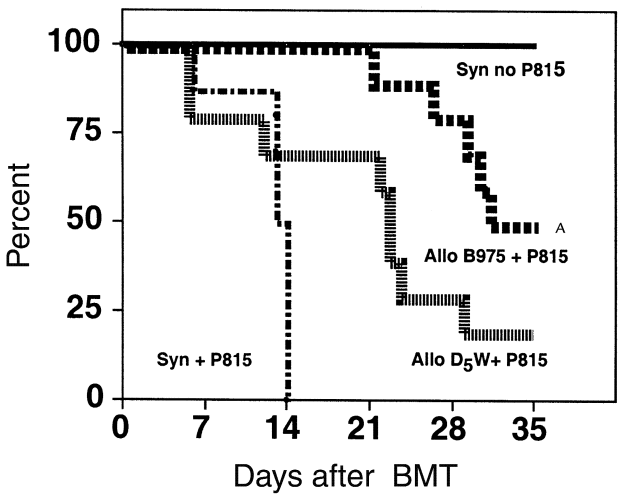

\section{Figure 5}

Treatment with B975 preserves GVL activity and improves leukemiafree survival. As shown, B6D2F1 mice received syngeneic $(n=8)$ or allogeneic BMT with B6Ly5.2 donors and either B975 $(n=10)$ or $\mathrm{D}_{5} \mathrm{~W}(n=10)$ as described in Figure 1. P815 cells $(2,000)$ were added to the BM inoculum at day 0 as described in Methods. Animals were monitored daily for survival, and necropsy was performed on all dying animals to determine whether cause of death was from leukemia or GVHD. ${ }^{A} P<0.05$; allogeneic + B975 vs. allogeneic + $D_{5}$ W. Syn no P815, syngeneic BMT without P815 cells.

importantly, their $\mathrm{T}$ cell responses to host antigens were equivalent (14). BMT with cells from donors resistant to the effects of LPS resulted in significantly less severe target organ and systemic GVHD (14).

Unfortunately, the toxicity of GVHD is difficult to separate from the desirable GVL effect, a well-recognized form of immunologically mediated tumor eradication; leukemia relapse rates after allogeneic BMT are inversely correlated to the presence and severity of $\operatorname{GVHD}(1,2,21)$. The physiology of the GVL effect is complex and is likely to involve multiple antitumor mechanisms involving both cellular and inflammatory effectors and various apoptotic pathways. The inflammatory cytokines released by LPS stimulation significantly contribute to the toxicity of GVHD but may have a more limited role in the eradication of residual leukemia, whereas donor cytotoxic T lymphocytes, natural killer cells, and lymphokineactivated cells are the principal mediators of GVL $(1,51)$. Indeed, the bulk of literature describing the principles of clinical GVL effects suggest that the induction of GVL activity depends upon the following: (a) the presence of cellular effectors, (b) the antigen specificity of these effectors (i.e., does the cell recognize antigens specific to the tumor, to host tissues, or both), and (c) their functional activity (i.e., the ability of an effector to kill a target once it is recognized) (52). GVL activity also depends upon the immunologic characteristics of the leukemia target that affects its susceptibility or resistance to GVL effector cells (51-53). This hypothesis is consistent with the observation that strategies of nonselective donor $\mathrm{T}$ cell depletion have failed to reduce GVHD severity without compromising GVL after clinical BMT $(21,22)$.

To determine whether administration of B975 could effectively separate GVHD from GVL effects after allogeneic BMT, we used an established mouse model of GVL 
activity wherein P815, a mastocytoma cell line, is added to the stem cell inoculum on day 0 . Although the use of tumor cell lines as models for malignancy is subject to several limitations, including selective pressures inherent in the long-term maintenance of such lines in vitro that may cause subtle but significant shifts in tumor cell biology compared with spontaneously occurring malignancies, such systems have been useful in elucidating effector mechanisms involved in GVL activity. We have found that allogeneic antitumor effects to P815 are dependent upon both $\mathrm{CD}^{+}$and $\mathrm{CD}^{+} \mathrm{T}$ cells and TNF- $\alpha$ and perforinmediated cytotoxicity $(23,43)$. These results are in accord with recent work by Tsukada and colleagues which showed that administration of a murine anti-TNF- $\alpha \mathrm{Ab}$ or BMT with perforin knockout $\mathrm{T}$ cells resulted in early tumor progression and death from P815 (54). P815 cells are MHC class I positive, class II negative, and Fas resistant. Thus, the requirement of $\mathrm{CD} 4^{+}$cells for GVL activity against P815 suggests that these cells are necessary not only for the activation and expansion of $\mathrm{CD}^{+}$effectors, but also for the secretion of inflammatory mediators, including TNF- $\alpha$, which contribute to GVHD induction and tumor eradication in this system $(11,23,43)$.

The data presented in this report are consistent with previous work where strategies directed toward protecting the GI tract and disrupting inflammatory cytokine cascades, while leaving cellular cytolytic function intact, have been successful in separating GVHD and GVL after experimental BMT $(23,25,27)$. Although LPS antagonism with B975 had no effect on donor $\mathrm{T}$ cell responses to host and tumor targets, we cannot rule out a contribution of persistent cytokine effector mechanisms to tumor eradication. The observation that $\mathrm{B} 975$ reduced, but did not totally abrogate serum TNF- $\alpha$ levels after allogeneic BMT may help to explain why GVL activity was preserved in this study, but was significantly reduced in the context of direct neutralization of TNF- $\alpha(43,54)$. In addition, it remains unresolved as to what effect, if any, LPS antagonism has on levels of membrane-bound TNF$\alpha$, which contribute to cell-mediated cytotoxicity and can also be effectively neutralized by mAb therapy (55). Finally, it is possible that the relative contribution of cellular versus cytokine effector mechanisms to P815 eradication is dependent upon the size of the tumor burden; the tumor cell inoculum used in the experiments by Tsukada et al. that highlighted the importance of TNF- $\alpha$ to GVL activity was 50 -fold greater than that injected in the current study $\left(1 \times 10^{5}\right.$ vs. $2 \times 10^{3} \mathrm{P} 815$ cells, respectively).

Taken together, our findings reveal a critical role for LPS in the early inflammatory events responsible for GI toxicity and subsequent mortality from GVHD; LPS acts via a positive feedback mechanism to amplify GI tract damage mediated by TNF- $\alpha$. These data suggest that a new class of pharmacologic agents, such as LPS antagonists, may serve as effective adjuncts to standard GVHD prophylaxis regimens. Acyl group modifications of originally developed compounds have led to improved sta- bility, solubility, and neutralizing capacity of current clinical candidates, which are now being studied in preparation for phase I and II trials for clinical sepsis syndrome $(56,57)$. The ability to use these molecules early in the time course of BMT may ultimately help clinicians reduce the toxicity of acute GVHD without compromising GVL activity or further suppressing donor immunologic reconstitution. Such novel strategies may therefore have promise in making allogeneic BMT a more safe and feasible option for a greater number of patients.

\section{Acknowledgments}

This work was supported by NIH grants HL-03565 and HL-55162 and by The J.P. McCarthy 1999 Developmental Grant Program. K.R. Cooke is a scholar of the National Marrow Donor Program and the Amy Strelzer-Manasevit Scholarship Program.

1. Truitt, R. 1995. Principles of graft-vs.-leukemia reactivity. Biol. Blood Marrow Transplant. 1:61-68.

2. Barrett, A.J., et al. 1992. Bone marrow transplantation for Philadelphia chromosome-positive acute lymphoblastic leukemia. Blood. 79:3067-3070.

3. Ferrara, J.L.M., and Deeg, H.J. 1991. Graft versus host disease. N. Engl. J. Med. 324:667-674.

4. Hill, G., Krenger, W., and Ferrara, J. 1998. Cytokine dysregulation in acute graft-versus-host disease. Hematology. 2:423-434.

5. Morrison, D., and Ryan, J. 1987. Endotoxin and disease mechanisms. Annu. Rev. Med. 38:417-432.

6. Raetz, C. 1990. Biochemistry of endotoxins. Annu. Rev. Biochem. 59:129-170.

7. Krenger, W., Hill, G., and Ferrara, J. 1997. Cytokine cascades in acute graft-versus-host disease. Transplantation. 64:553-558.

8. Kichian, K., Nestel, F.P., Kim, D., Ponka, P., and Lapp, W.S. 1996. IL-12 p40 messenger RNA expression in target organs during acute graft-versus-host disease. J. Immunol. 157:2851-2856.

9. Nestel, F.P., Price, K.S., Seemayer, T.A., and Lapp, W.S. 1992. Macrophage priming and lipopolysaccharide-triggered release of tumor necrosis factor alpha during graft-versus-host disease. J. Exp. Med. 175:405-413.

10. Hill, G., and Ferrara, J. 2000. The primacy of the gastrointestinal tract as a target organ of acute graft-versus-host disease: rationale for the use of cytokine shields in allogeneic bone marrow transplantation. Blood. 95:2754-2759.

11. Hill, G.R., et al. 1997. Total body irradiation effects on acute graft versus host disease. The role of gastrointestinal damage and inflammatory cytokines. Blood. 90:3204-3213.

12. Krenger, W., Snyder, K.M., Byon, C.H., Falzarano, G., and Ferrara, J.L.M. 1995. Polarized type 2 alloreactive CD4+ and CD8+ donor T cells fail to induce experimental acute graft-versus-host disease. J. Immunol. 155:585-593.

13. Cooke, K.R., et al. 1996. An experimental model of idiopathic pneumonia syndrome after bone marrow transplantation. I. The roles of minor $\mathrm{H}$ antigens and endotoxin. Blood. 8:3230-3239.

14. Cooke, K., et al. 1998. Tumor necrosis factor- $\alpha$ production to lipopolysaccharide stimulation by donor cells predicts the severity of experimental acute graft versus host disease. J. Clin. Invest. 102:1882-1891.

15. Heidt, P., and Vossen, J. 1992. Experimental and clinical gnotobiotics: influence of the microflora on graft-versus-host disease after allogeneic bone marrow transplantation. J. Med. 23:161-173.

16. van Bekkum, D., Roodenburg, J., Heidt, P., and van der Waaj, D. 1974. Mitigation of secondary disease of allogeneic mouse radiation chimeras by modification of the intestinal microflora. J. Natl. Cancer Inst. 52:401-406.

17. Storb, R., et al. 1983. Graft-versus-host disease and survival in patients with aplastic anemia treated by marrow grafts from HLA-identical siblings. Beneficial effect of a protective environment. N. Engl. J. Med. 308:302-307.

18. Moller, J., Skirhoj, P., Hoiby, N., and Peterson, F.B. 1982. Protection against graft versus host disease by gut sterilization? Exp. Hematol. 10:101-102.

19. Beelen, D.W., et al. 1992. Evidence that sustained growth suppression of intestinal anaerobic bacteria reduces the risk of acute graft-versus-host disease after sibling marrow transplantation. Blood. 80:2668-2676.

20. Beelen, D., Elmaagacli, A., Muller, K., Hirche, H., and Schaefer, U. 1999. 
Influence of intestinal bacterial decontamination using metronidazole and ciprofloxacin or ciprofloxacin alone on the development of acute graft-versus-host disease after marrow transplantation in patients with hematologic malignancies: final results and long term follow-up of an open-label prospective randomized trial. Blood. 93:3267-3275.

21. Horowitz, M.M., et al. 1990. Graft-versus-leukemia reactions after bone marrow transplantation. Blood. 75:55-562

22. Apperley, J., Mauro, F., and Goldman, J. 1988. Bone marrow transplantation for chronic myeloid leukemia in first chronic phase: importance of a graft-versus-leukemia effect. Br. J. Haematol. 69:239-245.

23. Teshima, T., et al. 1999. IL-11 separates graft-versus-leukemia effects from graft-versus-host disease after bone marrow transplantation. J. Clin Invest. 104:317-325.

24. Fowler, D.H., Kurasawa, K., Husebekk, A., Cohen, P.A., and Gress, R.E. 1994. Cells of the Th2 cytokine phenotype prevent LPS-induced lethality during murine graft-versus-host reaction. J. Immunol. 152:1004-1011.

25. Krijanovski, O., et al. 1999. Keratinocyte growth factor (KGF) separates graft versus leukemia effects from graft-versus-host disease. Blood. 94:825-831.

26. Christ, W., et al. 1995. E5531, a pure endotoxin antagonist of high potency. Science. 268:80-83.

27. Hill, G.R., et al. 1998. Interleukin-11 promotes T cell polarization and prevents acute graft-versus-host disease after allogeneic bone marrow transplantation. J. Clin. Invest. 102:115-123.

28. Fontaine, P., and Perreault, C. 1990. Diagnosis of graft-versus-host disease in mice transplanted across minor histocompatibility barriers. Transplantation. 49:1177-1179.

29. Piguet, P.F., Grau, G.E., Allet, B., and Vassalli, P.J. 1987. Tumor necrosis factor/cachectin is an effector of skin and gut lesions of the acute phase of graft-versus-host disease. J. Exp. Med. 166:1280-1289.

30. Hattori, K., et al. 1998. Differential effects of anti-Fas ligand and antitumor necrosis factor alpha antibodies on acute graft-versus-host disease pathologies. Blood. 91:4051-4055.

31. Crawford, J.M. 1997. Graft-versus-host disease of the liver. In Graft-ver sus-host disease. J.L.M. Ferrara, H.J. Deeg, and S.J. Burakoff, editors. Marcel Dekker Inc. New York, New York, USA. 315-336.

32. Billingham, R.E. 1966. The biology of graft-versus-host reactions. Harvey Lect. 62:21-78.

33. Vella, A.T., Mitchell, T., Groth, B., Linsley, P.S., and Green, J.M. 1997. CD28 engraftment and proinflammatory cytokines contribute to $T$ cell expansion and long-term survival in vivo. J. Immunol. 158:4714-4720.

34. Pape, K.A., Khoruts, A., Mondino, A., Jenkins, M.K. 1997. Inflammatory cytokines enhance the in vivo clonal expansion and differentiation of antigen-activated CD4+ T cells. J. Immunol. 159:591-598.

35. Tough, D.F., Sun, S., and Sprent, J. 1997. T cell stimulation in vivo by lipopolysaccharide (LPS). J. Exp. Med. 185:2089-2094.

36. Holler, E., et al. 1990. Increased serum levels of tumor necrosis factor alpha precede major complications of bone marrow transplantation. Blood. 75:1011-1016

37. Holler, E., et al. 1997. Inflammatory reactions induced by pretransplant conditioning: an alternative target for modulation of acute GVHD and complications following allogeneic bone marrow transplantation. Leuk. Lymphoma. 25:217-224.

38. Remberger, M., Ringden, O., and Markling, L. 1994. TNF-alpha levels are increased during bone marrow transplant conditioning in patients who develop acute GVHD. Bone Marrow Transplant. 15:99-104.

39. Mayer, F.R., et al. 1996. High response of TNF $\alpha$ secretion in vivo in patients undergoing BMT may be associated with the -308 bp TNFalpha-gene enhancer-polymorphism. Bone Marrow Transplant. 17(Suppl. 1):s101. (Abstr.)

40. Holler, E., et al. 1995. Modulation of acute graft-versus-host disease after allogeneic bone marrow transplantation by tumor necrosis factor alpha (TNF-alpha) release in the course of pretransplant conditioning: role of conditioning regimens and prophylactic application of a monoclonal antibody neutralizing human TNF-alpha (MAK 195F). Blood. 86:890-899.

41. Price, K.S., Nestel, F.P., and Lapp, W.S. 1997. Progressive accumulation of bacterial lipopolysaccharide in vivo during murine acute graft-versushost disease. Scand. J. Immunol. 45:294-300.

42. Nestel, F., et al. 1997. The role of endotoxin in the pathogenesis of acute graft-versus-host disease. In Graft-versus-host disease. 2nd edition. J.L.M. Ferrara, H.J. Deeg, and S.J. Burakoff, editors. Marcel Dekker Inc. New York, New York, USA. 501-524.

43. Hill, G., et al. 1999. Differential roles of IL-1 and TNF $\alpha$ on graft-versushost disease and graft versus leukemia. J. Clin. Invest. 104:459-467.

44. Fegan, C., Poynton, C., and Whittaker, J. 1990. The gut mucosal barrier in bone marrow transplantation. Bone Marrow Transplant. 5:373-377.

45. Poynton, C., Jackson, S., Fegan, C., Barnes, R., and Whittaker, J. 1992. Use of IgM enriched intravenous immunoglobulin (Pentaglobin) in bone marrow transplantation. Bone Marrow Transplant. 9:451-457.

46. Jackson, S.K., Parton, J., Barnes, R.A., Poynton, C.H., and Fegan, C. 1993. Effect of IgM-enriched intravenous immunoglobulin (pentaglobin) on endotoxemia and anti-endotoxin antibodies in bone marrow transplantation. Eur. J. Clin. Invest. 23:540-545.

47. van Bekkum, D.W., and Knaan, S. 1977. Role of bacterial microflora in development of intestinal lesions from graft versus host disease. J. Natl. Cancer Inst. 58:787-790.

48. Vossen, J., et al. 1990. Prevention of infection and graft-versus-host disease by suppression of intestinal microflora in children treated with allogeneic bone marrow transplantation. Eur. J. Clin. Microbiol. Infect. Dis. 9:14-23.

49. Cohen, J., et al. 1987. Antibody titers to a rough-mutant strain of Escherichia coli in patients undergoing allogeneic bone marrow transplantation. Lancet. 1:8-11.

50. Bayston, K., Baumgartner, J., Clark, P., and Cohen, J. 1991. Anti-endotoxin antibody for prevention of acute GVHD. Bone Marrow Transplant. 8:426-427.

51. Barrett, J., and Malkovska, V. 1996. The graft-versus-leukemia effect. Curr. Opin. Oncol. 8:89-95.

52. Truitt, R., Johnson, B., McCabe, C., and Weiler, M. 1997. Graft versus leukemia. In Graft-versus-host disease. 2nd Edition. J. Ferrara, H. Deeg, and S. Burakoff, editors. Marcel Dekker Inc. New York, New York, USA. 385-424.

53. Morecki, S., and Slavin, S. 2000. Toward amplification of a graft-versusleukemia effect while minimizing graft-versus-host disease. J. Hematother. Stem Cell Res. 9:355-366.

54. Tsukada, N., Kobata, T., Aizawa, Y., Yagita, H., and Okumura, K. 1999. Graft-versus-leukemia effect and graft-versus-host disease can be differentiated by cytotoxic mechanisms in a murine model of allogeneic bone marrow transplantation. Blood. 93:2738-2747.

55. Eissner, G., et al. 1995. Critical involvement of transmembrane tumor necrosis factor-alpha in endothelial programmed cell death mediated by ionizing radiation and bacterial endotoxin. Blood. 86:4184-4193.

56. Bunnell, E., et al. 2000. A lipid A analog, E5531, blocks the endotoxin response in human volunteers with experimental endotoxemia. Crit. Care Med. 28:2713-2720.

57. Rossignol, D., et al. 1999. Synthetic endotoxin antagonists. In Endotoxin in health and disease. H. Brade, D. Morrison, S. Opal, and S. Vogel, editors. Marcel Dekker Inc. New York, New York, USA. 699-717. 